\title{
The very reddened open clusters Pismis 23 (Lyngå 10) and Stephenson $2^{\star, \star \star}$
}

\author{
S. Ortolani ${ }^{1}$, E. Bica ${ }^{2}$, B. Barbuy ${ }^{3}$, and Y. Momany ${ }^{1}$ \\ 1 Università di Padova, Dept. di Astronomia, Vicolo dell'Osservatorio 2, 35122 Padova, Italy \\ e-mail: momany@pd.astro.it \\ ${ }^{2}$ Universidade Federal do Rio Grande do Sul, Dept. de Astronomia, CP 15051, Porto Alegre 91500-970, Brazil \\ e-mail: bica@if.ufrgs.br \\ 3 Universidade de São Paulo, CP 3386, São Paulo 01060-970, Brazil \\ e-mail: barbuy@astro.iag.usp.br
}

Received 15 March 2002 / Accepted 7 May 2002

\begin{abstract}
Pismis 23 and Stephenson 2 are two very reddened open clusters, projected close to the Galactic plane. We present $V I$ and $J H$ photometry for Pismis 23 and $I$ and Gunn $z$ for Stephenson 2.

We derive a relatively old age of $\sim 700 \mathrm{Myr}$ for Pismis 23 , with a reddening value of $E(B-V)=1.73$ and a distance $d_{\odot} \approx 2.6 \mathrm{kpc}$ from the Sun.

Stephenson 2 has a clump of red supergiants, indicating a young age of about $20 \mathrm{Myr}$, somewhat younger and more distant $\left(d_{\odot} \approx 6 \mathrm{kpc}\right)$ than recently reported in the literature. The age estimate for such a young object is crucial for the distance estimate. The high reddening of these two clusters relative to predictions from the diffuse interstellar medium at their distances is due to discrete intervening clouds.
\end{abstract}

Key words. open clusters and associations: individual: Pismis 23, Stephenson 2 - stars: Hertzsprung-Russell (HR) and C-M diagrams

\section{Introduction}

In order to obtain relevant Colour-Colour and ColourMagnitude Diagrams (CMD) a suitable depth in each band is needed, and large amounts of reddening can impose a detection threshold in certain bandpasses.

Among globular clusters, a few examples of limiting cases of optical detections are Liller 1 and UKS 1 . Their Red Giant Branches (RGB) could be seen only in a $I$ vs. Gunn $z$ diagram (Ortolani et al. 1996, 1997). From this point of view they might be considered infrared clusters. In order to assess full information on their properties, much deeper diagrams were obtained with the Hubble Space Telescope using NICMOS in $J$ and $H$ (Ortolani et al. 2001). On the other hand, 2MASS-GC1 and 2MASS-GC2 required infrared bands both for identification and CMD studies (Hurt et al. 2000; Ivanov et al. 2000).

Send offprint requests to: $\mathrm{B}$. Barbuy,

e-mail: ortolani@pd.astro.it

* Observations collected at the European Southern Observatory ESO, Chile.

$\star \star$ Tables 2 and 3 are only available in electronic form at the CDS via anonymous ftp to cdsarc.u-strasbg.fr (130.79.128.5) or via

http://cdsweb.u-strasbg.fr/cgi-bin/qcat?]/A+A/390/931
Regarding open clusters, one example of a limiting case of extreme reddening, but where the cluster upper main sequence (MS) can still be studied in a $V$ vs. $V-I$ diagram, is Westerlund 1 (Piatti et al. 1998).

In the present study, we analyse two open clusters that are very reddened: they are undetectable in sky survey $B$ plates, and whereas Pismis 23 is clear in $R$, Stephenson 2 is only evident in infrared bands. Pismis 23 is a compact open cluster and Stephenson 2 is a loose one.

Pismis (1959) identified the cluster Pismis 23 on Schmidt plates at the Tonantzintla Observatory. It was also reported in 1963 by G. Lyngå (Lyngå 10), as a private communication to Alter et al. (1970). In the latter compilation it is designated also as $\mathrm{OCl}-967$ and C1620-488. More recent denominations are BH 190 ou vdBH 190 (van den Bergh \& Hagen 1975), and ESO 226-SC5 (Lauberts 1982). It is located in Norma, at J2000.0 $\alpha=16^{\mathrm{h}} 23^{\mathrm{m}} 58 \mathrm{~s}$ and $\delta=-48^{\circ} 53^{\prime} 33^{\prime \prime}\left(l=334.66^{\circ}, b=0.42^{\circ}\right)$. It has an approximate dimension of $1.5^{\prime}$ on the $R$ image of the Digital Sky Survey (XDSS).

Stephenson (1990) reported a candidate open cluster, identified from near-infrared photographic plates obtained at the Burrell Schmidt telescope, with an objective prism. He identified a concentration of $10 \mathrm{M}$ supergiants. We suggest to refer to this cluster as Stephenson 2, since in an earlier study 
Stephenson (1959) identified another cluster, given in subsequent open cluster catalogues (e.g. Alter et al. 1970). Recently, Nakaya et al. (2001) studied the present cluster, by means of $V$, $R, I, J, H$ and $K$ photometry. The cluster is difficult to be seen in $I$, owing to patchy absorption, but it becomes clear in $J, H$ and $K$. Besides the red supergiants, there are upper MS stars. Nakaya et al. obtain $A_{V}=11.2$ and a distance of $d_{\odot} \approx 1.5 \mathrm{kpc}$ from the Sun.

Stephenson 2 is located in Scutum at $\mathrm{J} 2000.0 \alpha=$ $18^{\mathrm{h}} 39^{\mathrm{m}} 20^{\mathrm{s}}, \delta=-6^{\circ} 01^{\prime} 44^{\prime \prime}, l=26.18^{\circ}, b=-0.06^{\mathrm{o}}$.

In the present study, we discuss CMDs of Stephenson 2 in $I$ vs. $I-$ Gunn $z$. For Pismis 23, for the first time CMDs are presented, and the cluster parameters are derived.

In Sect. 2 the observations are described. In Sects. 3 and 4 we discuss respectively Pismis 23 and Stephenson 2. Concluding remarks are given in Sect. 5.

\section{Observations and reductions}

Pismis 23 was observed in the optical, on 2000 March 5, at the Danish $1.54 \mathrm{~m}$ telescope at the European Southern Observatory, using the DFOSC camera together with a CCD Loral/Lesser of $2052 \times 2052$ pixels, with pixel size $15 \mu \mathrm{m}$. It corresponds to $0.39^{\prime \prime}$ on the sky. The full field is $13.7^{\prime} \times 13.7^{\prime}$.

It was also observed in the infrared $J$ and $H$ colours at the New Technology Telescope NTT-ESO, with SOFI and the detector Hawaii $\mathrm{HgCdTe}$ of $1024 \times 1024$ pixels, with pixel size $18.5 \mu \mathrm{m}$, used in wide field imaging mode, giving $0.29^{\prime \prime} /$ pixel, and a field of $4.9^{\prime} \times 4.9^{\prime}$. Four sets of 30 co-added images have been obtained with $2 \mathrm{~s}$ exposure per colour. The sky was obtained in offset mode with the same exposure times.

We show in Fig. 1 a Danish I image of Pismis 23, in an extraction of dimensions $\approx 7.5^{\prime} \times 7.5^{\prime}$. The cluster is compact and rather dense, towards a rich field.

Stephenson 2 was observed also at the Danish $1.54 \mathrm{~m}$ telescope, on 1994 May 19, using the Tektronix CCD ESO \# 28, of $1024 \times 1024$ pixels, with pixel size $24 \mu \mathrm{m}\left(0.37^{\prime \prime}\right.$ on the sky). The full field is $6.3^{\prime} \times 6.3^{\prime}$.

The log of observations is given in Table 1 .

In view of making available the data for future proper motion studies, in Table 2 are given $V, I$ colours and $X, Y$ coordinates of Pismis 23, and in Table 3 are given $I$ and $X, Y$ coordinates of Stephenson 2, available in electronic form at the CDS-Strasbourg datacenter.

The stellar photometry was carried out with the DAOPHOT II code in MIDAS environment. Landolt (1983, 1992) standard stars were used for the calibrations.

The reductions of Pismis $23 \mathrm{~V}$ and $I$ were carried out in the standard way, and the calibration equations are:

$$
\begin{aligned}
V & =26.56+0.01(V-I)+v \\
I & =24.61+i
\end{aligned}
$$

where coefficients are for $10 \mathrm{~s}$ and $5 \mathrm{~s}$ exposures respectively and airmass of 1.1 .

The NTT $J$ and $H$ photometry has been derived following the standard procedure for near IR images. The sky (plus bias) was subtracted using the star filtered images obtained from four

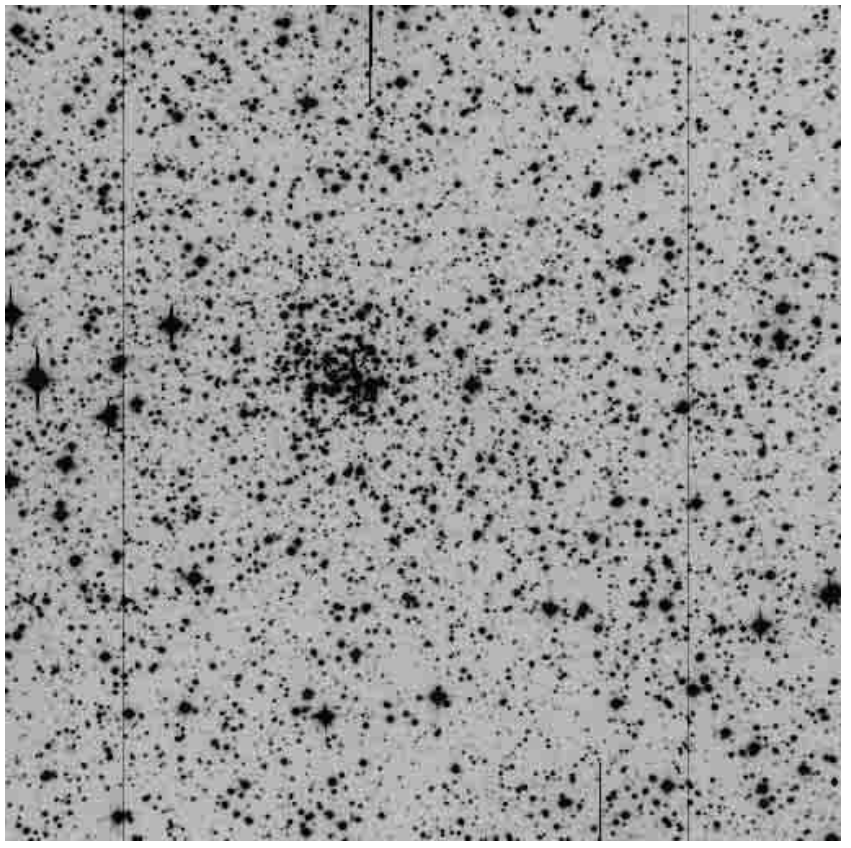

Fig. 1. $I$ image $(10 \mathrm{~min})$ of Pismis 23 . Extraction of dimensions $\approx$ $7.5^{\prime} \times 7.5^{\prime}$. North is at the top and east to the left.

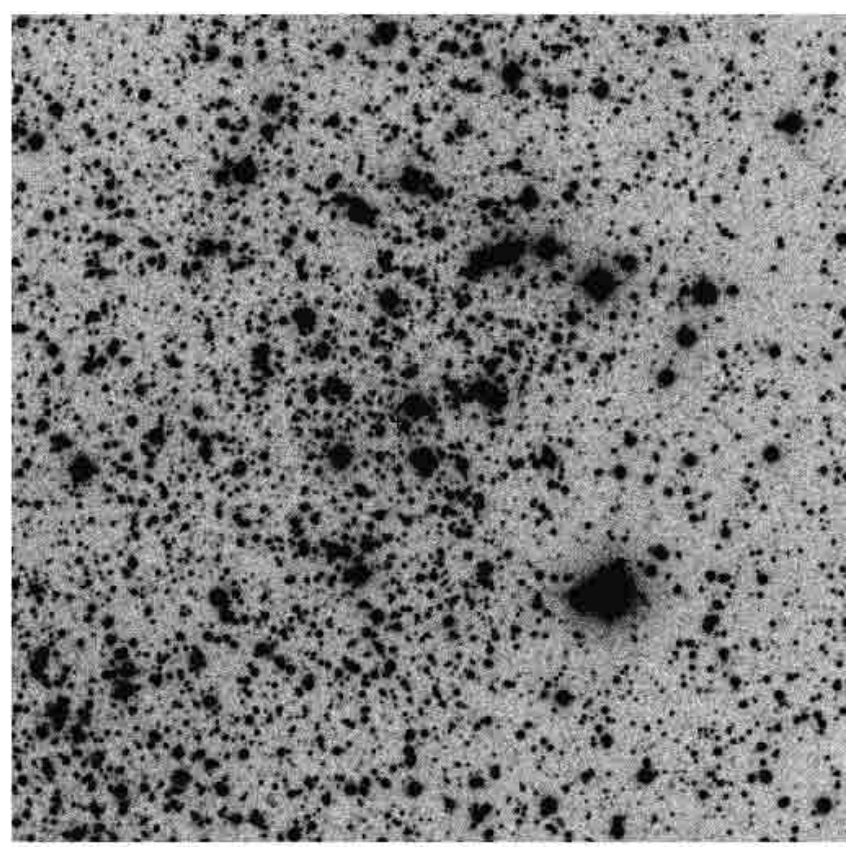

Fig. 2. I image (3 min) of Stephenson 2. Dimensions are $6.3^{\prime} \times 6.3^{\prime}$. North is up and east to the left.

shifted images in each colour, on the offset sky. High signalto-noise flatfields (superflat) available in ESO webpages have been used for the flatfielding. The bias, sky and flatfield corrected images, have been processed using the DAOPHOT II package available in MIDAS. Six standard stars from Elias et al. (1982) have been observed during the night for the calibration. The derived constants are $K_{J}=22.99 \pm 0.01, K_{H}=$ $22.90 \pm 0.015, K_{K}=22.36 \pm 0.02$ at 1.1 airmasses for $1 \mathrm{~s}$ exposure. In spite of the small dispersion of the zero-points, the comparison of the targets, taken in different positions on 
Table 1. Log of observations.

\begin{tabular}{|c|c|c|c|c|c|}
\hline Target & Filter & Date & Tel. & $\begin{array}{l}\text { Exp. } \\
\text { (s.) }\end{array}$ & $\begin{array}{l}\text { Seeing } \\
\left({ }^{\prime \prime}\right)\end{array}$ \\
\hline \multirow[t]{6}{*}{ Pismis 23} & V & 05.03 .00 & Danish & 60 & 1.4 \\
\hline & $I$ & $"$ & $"$ & 30 & 1.4 \\
\hline & $I$ & $"$ & " & 600 & 1.0 \\
\hline & V & $"$ & " & 1200 & 1.3 \\
\hline & $J$ & 02.07 .00 & NTT & 240 & 1.5 \\
\hline & $H$ & $"$ & $"$ & 240 & 1.5 \\
\hline \multirow[t]{2}{*}{ Stephenson 2} & I & 19.05 .94 & Danish & 180 & 1.5 \\
\hline & Gunn $z$ & $"$ & $"$ & 60 & 1.5 \\
\hline
\end{tabular}

the sky during the same night, indicates a scatter of about \pm 0.2 magnitudes. We conclude that the night was possibly affected by cirrus. Therefore our $J, H$ photometry will be used for nonabsolute photometry purposes.

For the Danish observation of Stephenson 2, the calibration equation of the $I$ band is:

$I=26.095+i$

where coefficients are for $30 \mathrm{~s}$ exposures and airmass of 1.1. Gunn $z$ is uncalibrated.

Zero point uncertainties are dominant as sources of errors in all calibrations. It is typically of $\pm 0.03 \mathrm{mag}$. The magnitude transfer from the cluster images to the standard stars can amount to $0.03 \mathrm{mag}$. The internal errors are constant around 0.02 mag up to $I \approx 17$, increasing for fainter magnitudes.

\section{Pismis 23}

\subsection{Colour-magnitude diagrams}

In Fig. 3 we show the $V$ vs. $V-I$ diagram corresponding to a field extraction of $7.5^{\prime} \times 7.5^{\prime}$. A prominent field main sequence (MS) affected by a strong differential reddening is seen. Figure 4 shows the NTT full field $\left(4.9^{\prime} \times 4.9^{\prime}\right)$ in $J$ vs. $J-H$, where the sequences are better defined relative to Fig. 3; in particular giants are present at $J-H \approx 1.1$. The cluster is not evident in any of these large field CMDs (Figs. 3 and 4).

In Fig. 5 a NTT $J$ vs. $J-H$ extraction of $r<0.63^{\prime}(r<130$ pixels), centered on Pismis 23 is given. The cluster sequences are evident in this extraction, showing a tight well-populated MS and a few giants at $J \approx 11.5$.

In Fig. 6 a $V$ vs. $V-I$ CMD for the same spatial extraction as Fig. 5 is presented, with $r<95$ pixels. The cluster MS is much redder than the field MS (see Fig. 3).

In the $V$ vs. $V-I$ CMD (Fig. 6) the giants are nearly at the same level as the turnoff, in contrast with their much brighter location in the $J$ vs. $J-H$ (Fig. 5) with respect to the turnoff.

\subsection{Age}

In Figs. 5 and 6 solar metallicity Padova isochrones of $500 \mathrm{Myr}$ (Bertelli et al. 1994) are superimposed on the CMDs. Fits for a series of ages were tried, due to the difficulty that the giants

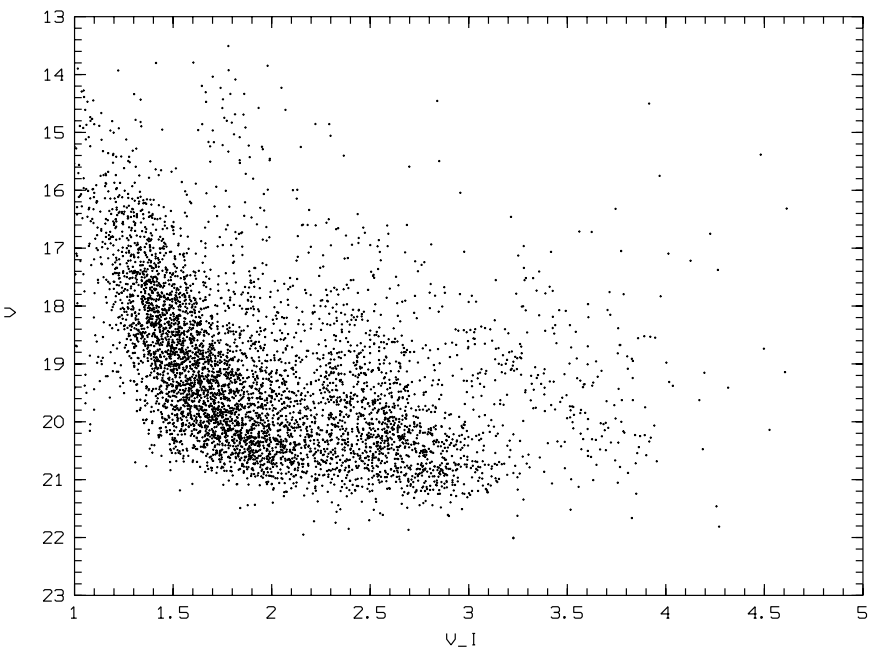

Fig. 3. $V$ vs. $V-I$ field diagram including Pismis 23. Extraction of dimensions $\approx 7.5^{\prime} \times 7.5^{\prime}$

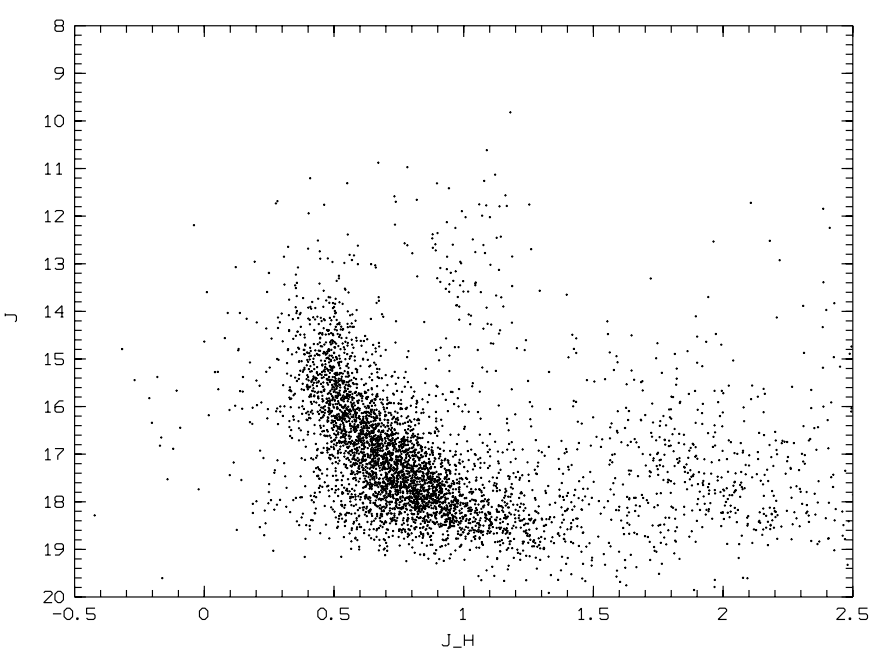

Fig. 4. NTT $J$ vs. $J-H$ diagram of full field including Pismis 23 . Extraction of dimensions $\approx 4.9^{\prime} \times 4.9^{\prime}$.

are too few, and up to $1 \mathrm{Gyr}$ the fit is still acceptable. We adopt $700 \pm 200 \mathrm{Myr}$ as a best match.

\subsection{Reddening and distance}

From Fig. 6 a difference $\Delta V=17.4$ and $\Delta(V-I)=2.30$ between the observed sequences and the isochrone are obtained.

Assuming a ratio $E(V-I) / E(B-V)=1.33$ (Dean et al. 1978 ), and $R=3.1$, we get $E(B-V)=1.73$ and $A_{V}=5.36$. The high reddening of this intermediate age cluster, therefore with no significant internal dust, is in part probably due to two dust clouds FeSt1-322 (Feitzinger \& Stüwe 1984) and HMSTG335.1+0.2 (Hartley et al. 1986).

An absolute distance modulus $(m-M)_{0}=12.04$ and a distance from the Sun $d_{\odot}=2.6 \mathrm{kpc}$ are derived. This is a relatively close cluster with a high reddening.

The Galactocentric coordinates are $X=-5.65(X<0$ means our side of the Galaxy), $Y=-1.11$ and $Z=0.02 \mathrm{kpc}$, considering the distance to the Galactic center to be $R=8 \mathrm{kpc}$ (Reid 1993). The cluster is located inside the solar circle. 


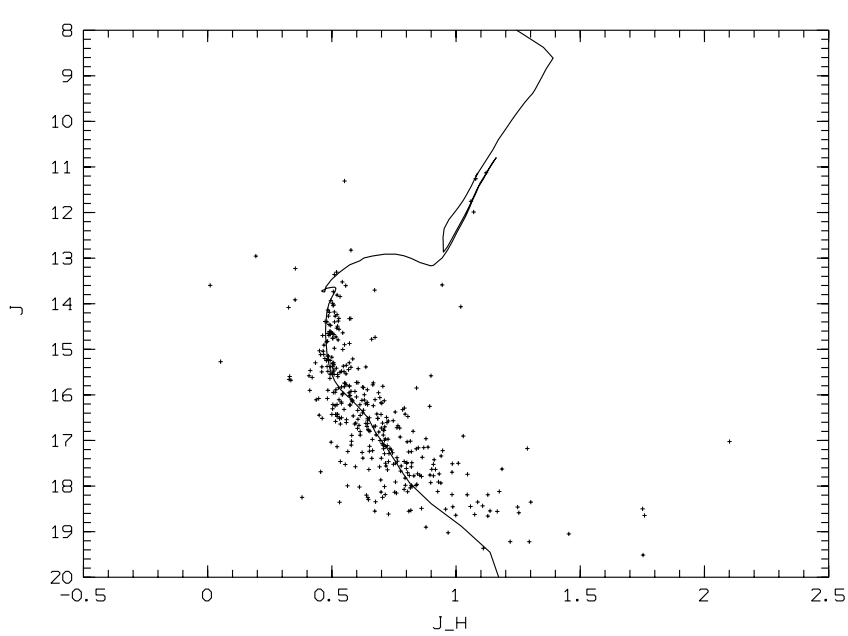

Fig. 5. Pismis 23: $J$ vs. $J-H$ extraction of $r<0.63^{\prime}$ with Padova isochrone of $500 \mathrm{Myr}$ overplotted.

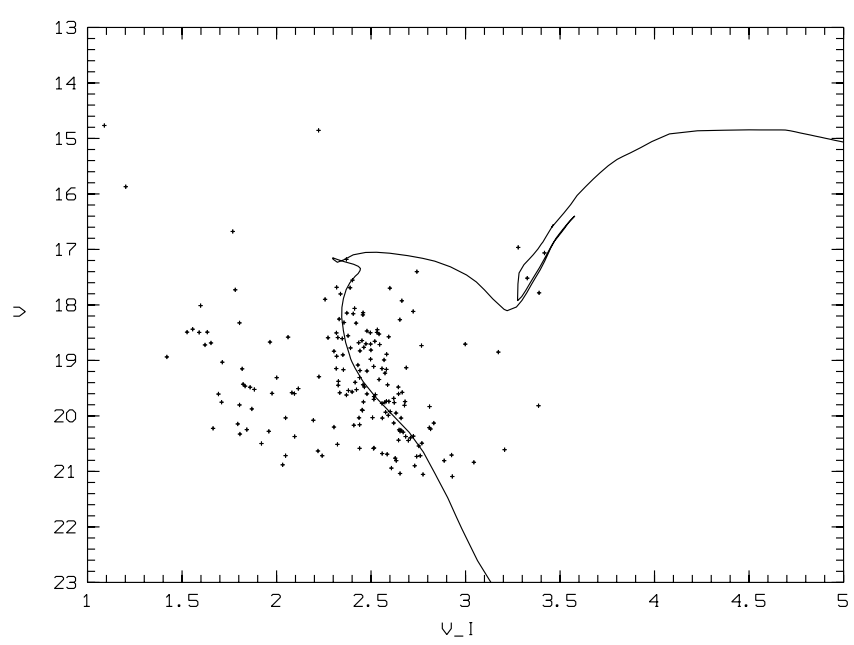

Fig. 6. Pismis 23: $V$ vs. $V-I$ extraction of $r<0.63^{\prime}$ with Padova isochrone of $500 \mathrm{Myr}$ overplotted.

\section{Stephenson 2}

The images of Nakaya et al. (2001), together with the present ones, indicate a cluster of diameter around $4^{\prime}$.

Figure 7 shows a $I$ vs. $I-$ Gunn $z$ for the whole field, centered on the object. The clump of red supergiants is obvious, together with two main sequences. In Fig. 8 the field corresponding to $r>2.5^{\prime}$ ( $r>400$ pixels) shows that the redder sequence is weakened with respect to the bluer one, making evident that the latter corresponds to the field foreground disk, and the redder one is the cluster MS, in agreement with the Nakaya et al. analysis. In Fig. 9 an extraction of radius $r<1.85^{\prime}$ $(r<300$ pixels) is given, showing that the two main sequences are comparable in number of stars, which shows that contamination is important even in central parts. The red supergiants are concentrated in the central parts (Fig. 9), confirming results from Stephenson (1990) and Nakaya et al. (2001). The high extinction of $A_{V}=11.2$ (Nakaya et al. 2001) and the colour separation between the bulk of the field MS and the cluster MS (Fig. 9) indicates that an intervening dust layer is present, probably corresponding to the dark cloud LDN 515 (Lynds 1962).

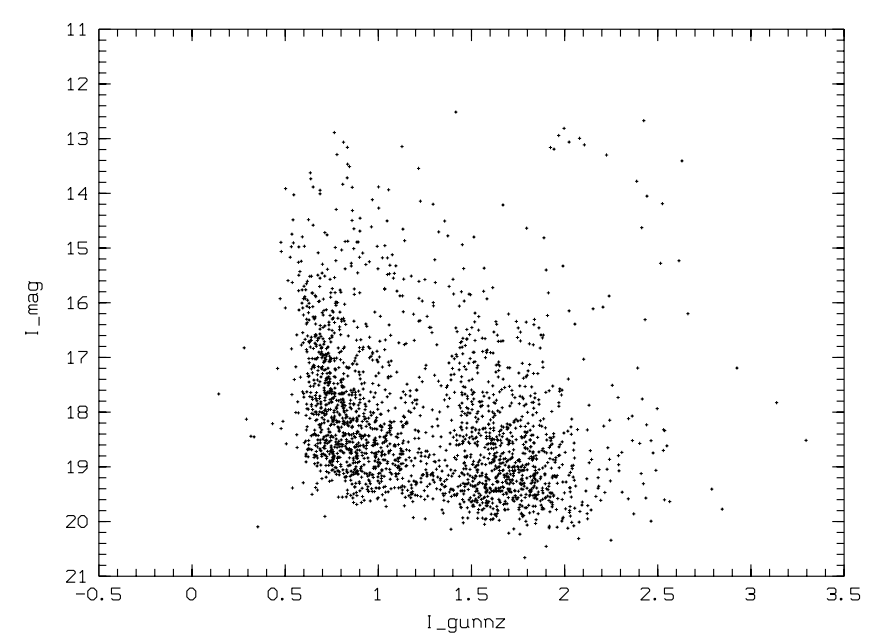

Fig. 7. Full field of Stephenson 2 in $I$ vs. $I-$ Gunn $z$.

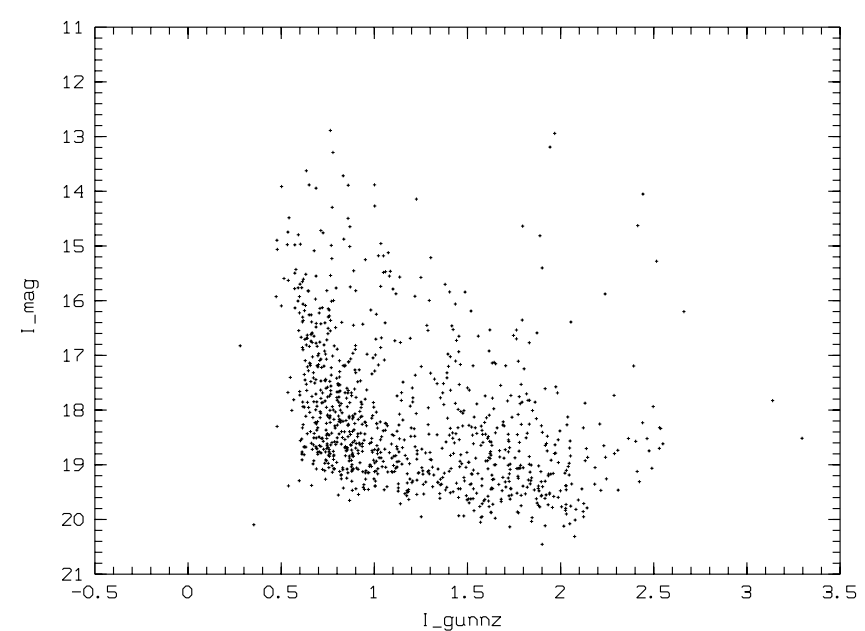

Fig. 8. Field surrounding Stephenson 2: $I$ vs. $I-$ Gunn $z$ extraction of $r>2.5^{\prime}$.

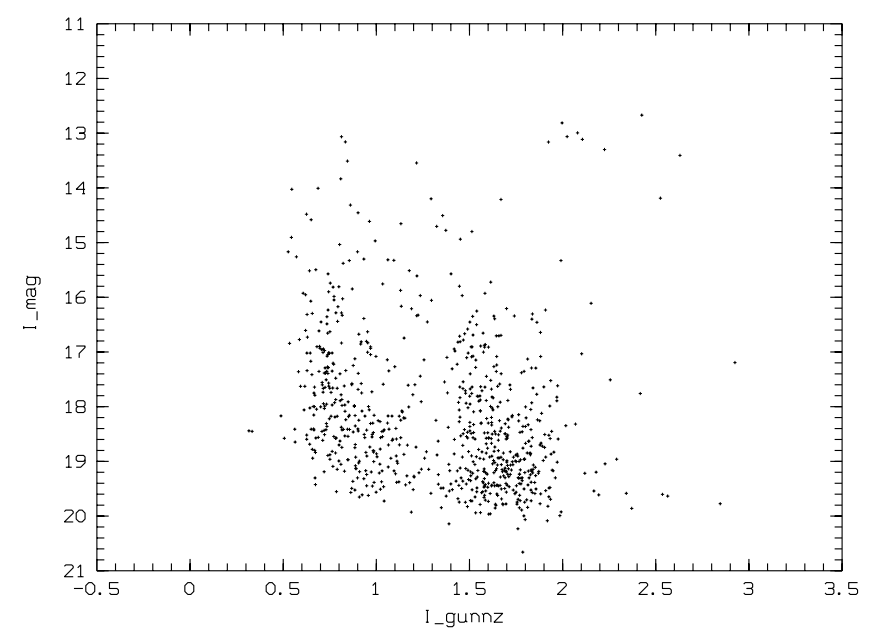

Fig. 9. Stephenson 2: $I$ vs. $I-$ Gunn $z$ for an extraction of $r<1.85^{\prime}$.

Red supergiant clumps occur typically at ages of 10$20 \mathrm{Myr}$, as in NGC 2004 of the LMC (Bica et al. 1990 and references therein), and as can be verified in the composite CMDs for Galactic open clusters given in terms of age groups by Mermilliod (1981a). Red supergiant clumps occur in the CMDs 
of NGC 884 and NGC 3766 groups of ages 12 and 22 Myr respectively (see Mermilliod 1981b and WEBDA database obswww. unige.ch/webda/navigation.html). This shows in which age range it is probable to find such stars. The clump seen in Fig. 9 suggests that the age of Stephenson 2 is around 10-20 Myr.

We verified from the Padova isochrones (Bertelli et al. 1994) the absolute $I$ magnitude $M_{I}$ of red supergiants corresponding to these ages. For $10 \mathrm{Myr}, M_{I}=-8.1$, and for $20 \mathrm{Myr}$, $M_{I}=-7.2$. In Stephenson 2 the clump is located at $I=13.0$ (Fig. 9). Adopting the reddening $A_{V}=11.2$ by Nakaya et al. (2001), then $A_{I}=6.35$, using $A_{I} / A_{V}=0.594$ (Schlegel et al. 1998).

A distance modulus $(m-M)_{0}=14.75$ and a distance of $d_{\odot}=8.9 \mathrm{kpc}$ were obtained for an age of $10 \mathrm{Myr}$, and $(m-M)_{0}=13.85$ and a distance of $d_{\odot}=5.9 \mathrm{kpc}$ for $20 \mathrm{Myr}$. This shows how dependent the distance determination is on the luminosity of the red supergiants with age. An age closer to $20 \mathrm{Myr}$ and a distance around $6 \mathrm{kpc}$ appear more likely. This is younger than the $50 \mathrm{Myr}$ and the distance is larger than given by Nakaya et al. (2001). The $I$ magnitudes of the supergiants and early type star candidates are compatible between this work and Nakaya et al. (2001). The difference in age and distance comes from the weight given by Nakaya et al. to the bright red stars in the isochrone fitting. Nakaya et al. interpreted these stars as massive giants, whereas in the present study we interpreted these as helium-burning stars in the isochrone fitting, similarly to the suggestion by Stephenson (1990).

\section{Conclusions}

We carried out photometry of the open clusters Pismis 23 and Stephenson 2, which are extremely reddened and difficult to study in the optical. For Pismis $23 \mathrm{VI}$ and $J H$ photometry were presented and $J H$ diagrams and $I$ and Gunn $z$ were used for Stephenson 2.

We derive $\sim 700 \mathrm{Myr}$ for Pismis 23, and $20 \mathrm{Myr}$ for Stephenson 2. A reddening value of $E(B-V)=1.73$ and $d_{\odot} \approx 2.6 \mathrm{kpc}$ from the Sun were derived for Pismis 23 . A distance of $d_{\odot} \approx 6 \mathrm{kpc}$ is obtained for Stephenson 2 .

In the diffuse interstellar medium, the extinction is typically of $A_{V}=1.5 \mathrm{mag} / \mathrm{kpc}$ (Méndez \& van Altena 1998). Lyngå (1982) obtained a lower value of $A_{V}=0.75 \mathrm{mag} / \mathrm{kpc}$ from open clusters. In the directions of Pismis 23 and Stephenson 2 values of about $A_{V}=2.1$ and $1.9 \mathrm{mag} / \mathrm{kpc}$ respectively are obtained. These higher values are caused by discrete clouds. Pismis 23 coincides with the Norma-Scutum arm (Taylor \& Cordes 1993), whereas Stephenson 2 may be more internal (uncertainties in distance are large for this cluster). In the case of
Pismis 23, the intervening clouds are in the Sagittarius-Carina arm as can be seen in the maps of Neckel et al. (1980). The direction of Stephenson 2 was not sampled in Neckel et al. (1980). Probably the dark clouds are due to the SagittariusCarina and Norma-Scutum itself.

Other very reddened open clusters should be used as probes of the interstellar medium characteristics.

Acknowledgements. We acknowledge partial financial support from the Brazilian agencies Fapesp and $\mathrm{CNPq}$, and the Italian Ministero dell'Università e della Ricerca Scientifica e Tecnologica (MURST) under the program on "Stellar Dynamics and Stellar Evolution in Globular Clusters: a Challenge for New Astronomical Instruments".

\section{References}

Alter, G., Ruprecht, J., \& Vanysek, V. 1970, Catalog of Star Clusters and Associations, 2nd edition, ed. G. Alter, B. Balazs, \& J. Ruprecht (Akademiai Kiado, Budapest)

Bertelli, G., Bressan, A., Chiosi, C., Fagotto, F., \& Nasi, E. 1994, A\&AS, 106, 275

Bica, E., Alloin, D., \& Santos, J. F. C. 1990, A\&A, 235, 103

Dean, J. F., Warren, P. R., \& Cousins, A. J. 1978, MNRAS, 183, 569

Elias, J. H., Frogel, J. A., Matthews, K., \& Neugebauer, G. 1982, AJ, 87,1029

Feitzinger, J. V., \& Stüwe, J. A. 1984, A\&AS, 58, 365

Hartley, M., Tritton, S. B., Manchester, R. N., Smith, R. M., \& Goss, W. M. 1986, A\&AS, 63, 27

Hurt, R. L., Jarrett, T. H., Kirkpatrick, J. D., et al. 2000, AJ, 120, 1876

Ivanov, V. D., Borissova, J., \& Vanzi, L. 2000, A\&A, 362, L1

Landolt, A. U. 1983, AJ, 88, 439

Landolt, A. U. 1992, AJ, 104, 340

Lauberts, A. 1982, ESO/Uppsala Survey of the ESO(B) atlas, Garching bei München, ESO

Lynds, B. T. 1962, ApJS, 7, 1

Lyngå, G. 1982, A\&A, 109, 213

Méndez, R. A., \& van Altena, W. F. 1998, A\&A, 330, 910

Mermilliod, J.-C. 1981a, A\&AS, 44, 467

Mermilliod, J.-C. 1981b, A\&A, 97, 235

Nakaya, H., Watanabe, M., Ando, M., Nagata, T., \& Sato, S. 2001, AJ, 122,876

Neckel, Th., Klare, G., \& Sarcander, M. 1980, A\&AS, 42, 251

Ortolani, S., Bica, E., \& Barbuy, B. 1996, A\&A, 306, 134

Ortolani, S., Bica, E., \& Barbuy, B. 1997, A\&AS, 126, 319

Ortolani, S., Barbuy, B., Bica, E., et al. 2001, A\&A, 376, 878

Piatti, A. E., Bica, E., \& Clariá, J. J. 1998, A\&AS, 127, 423

Pismis, P. 1959, BOTT 2, part 18, 37

Reid, M. J. 1993, ARA\&A, 31, 345

Schlegel, D. J., Finkbeiner, D. P., \& Davis, M. 1998, ApJ, 500, 525

Stephenson, C. B. 1959, PASP, 71, 145

Stephenson, C. B. 1990, AJ, 99, 1867

Taylor, J. H., \& Cordes, J. M. 1993, ApJ, 411, 674

van den Bergh, S., \& Hagen, L. 1975, AJ, 80, 11 